\title{
Sperm Protein Associated With the Nucleus on the X Chromosome N5
}

National Cancer Institute

\section{Source}

National Cancer Institute. Sperm Protein Associated With the Nucleus on the X

Chromosome N5. NCl Thesaurus. Code C93003.

Sperm protein associated with the nucleus on the X chromosome N5 (72 aa, $\sim 8 \mathrm{kDa}$ ) is encoded by the human SPANXN5 gene. This protein may be involved in the differentiation of sperm. 